\title{
The Efficiency of a $p$-n Solar Diode as a Function of the Recombination Velocity within the Depletion Layer
}

\author{
Mohamed K. El-Adawi ${ }^{1}$, Najla S. Al-Shameri ${ }^{2}$ \\ ${ }^{1}$ Physics Department, Faculty of Education, Ain Shams University, Cairo, Egypt \\ ${ }^{2}$ Physics Department, College of Science for Girls, Dammam University, Dammam, KSA \\ Email: adawish1@hotmail.com
}

Received September 14, 2012; revised October 16, 2012; accepted October 30, 2012

\begin{abstract}
The role of the carrier's recombination velocity $s_{i}$ within the depletion Layer of $p-n$ junction solar cell and the external bias voltage $V_{a}$ across the junction in determining the current density " $J$ " through the cell is revealed. The unsteady carrier diffusion equation is solved under illumination conditions considering a source spectral function $G(\lambda)$. The efficiency of the device as a function of $s_{i}, V_{a}, G(\lambda)$ is obtained. Computations considering a silicon solar cell are given as an illustrative example.
\end{abstract}

Keywords: Efficiency; Diffusion Equation; Recombination Velocity

\section{Introduction}

The performance of a solar cell has aroused the interest of many investigators [1-17]. The solar $p-n$ cell is a semiconductor photovoltaic cell. It is a passive transducer. It is fabricated such that one region is more highly doped denoted by $n^{+}$or $p^{+}$region. The density of the charge carriers in the highly doped region may be of three orders larger than the other region. This surface is usually subjected to the incident illuminations (emitter region). Due to large gradients, diffusion of charge carriers take place, holes from $P^{+}$region diffuse to the $\mathrm{n}$ region, while electrons diffuse from $\mathrm{n}$-region to $P^{+}$ region. As a result a depletion layer is formed on both sides of the contact metallurgical surface between both sides. This layer is of small thickness $(\cong 1 \mu \mathrm{m})$ and is called as the space-charge region (SCR). This layer contains the positive ions of the donner atoms on one side of the contact surface and the negative ions of the acceptor atoms on the other side, and thus an electric field is built and one gets what is called the built-in-voltage $\left(V_{b i}\right)$. It is also called the contact potential or the barrier potential. The direction of the electric field within the depletion layer will be from the N-region to the P-region.

At equilibrium the net current (the sum of the diffusion currents and the drift currents) through the depletion layer must be zero. Now if the cell is subjected to an external reverse voltage bias, the established external field will strengthen the internal one, and less current density passes through the depletion layer and vice versa [18]. This bias voltage disturbs the thermal equilibrium state of the device. When light strikes the highly doped surface, the absorbed quanta excite the bound electrons and electron-hole pairs are produced that move in all directions within the crystal [2]. The electron-hole generation process is accompanied with recombination processes. Now if the bias voltage is switched off, the builtin-voltage will change towards its equilibrium value, through a recombination process between the electrons and the holes during the decay of the external voltage [3]. The equilibrium state will be restored within a time interval $\tau$ called as the relaxation time or it is defined as the minority carrier life time .It is worth to note that the electric potential across the depletion layer controls its electric resistance [4]. Different factors affect the charge transport properties within the cell, such as the electric potential across the device, the recombination velocity between the charge carriers. The most important factor is the doping level $[5,6]$. This factor can be controlled through the fabrication technology. These factors affect the current density passing through the cell and thus change its efficiency. Different trials are oriented to study the performance of the solar cell through the study of the recombination velocity between the charge carriers.

With this respect one finds in principal two trends namely: 
1) The first trend does accept the mechanism where recombination occurs principally at one end surface of the semiconductor device (usually it is the highly doped one). In such a case the coordinate of such a surface is taken as the origin $x=0$, and a boundary condition expressing the charge continuity at this surface is given [18]. Such a trend accepts also the study of this performance considering the collection efficiency of the base, the emitter and the depletion layer [1,2,7-12]. It is shown that the normalized surface recombination velocity depends mainly on two parameters; these are the normalized scanning range and the normalized depth of the generation volume (that is subjected to the incident light).

2) The second trend accepts the mechanism where an effective interface recombination velocity $s_{i}$ that occurs principally within the depletion layer at the metallurgical interface $[3,4,13,14]$. Indeed, the effect of the characteristics of the space charge region and the processes taking place within this layer has rarely been considered [3,9]. El-Adawi et al. [15] studied theoretically the characteristics of the depletion layer. As a result, the dependence of its thickness and capacity on the doping ratio and the applied bias voltage is revealed. It is worth to note that a restriction on the doping ratio $\frac{N_{D}}{N_{A}}$ is also obtained [15]. A novel method [16] to estimate experimentally the thickness of the depletion layer $(\cong 6.7 \mu \mathrm{m})$ is introduced.

The main objective of the present article is to establish a relation between the recombination velocity at the metallurgical interface within the depletion layer and the current density which in turn affects the efficiency of the solar cell. The unsteady carrier diffusion equation is solved under illumination conditions.

\section{Derivation of the Basic Equations}

Consider a $p^{+}-n$ solar device (Figure 1), subjected to incident solar flux $\phi(\lambda)$ and a bias voltage $V_{a}$. For the unsteady state, the diffusion equation (after switching off the bias voltage) is written for the minor charge carriers in the form:

$$
\begin{aligned}
\nabla^{2}\left(n(x, t)-n_{0}\right)= & \frac{1}{D_{n}} \frac{\partial\left(n(x, t)-n_{0}\right)}{\partial t} \\
& +\frac{n(x, t)-n_{0}}{D_{n} \tau}-\frac{G(\lambda, x)}{D_{n}}
\end{aligned}
$$

where

$n(x, t)$, the concentration of the minor charge carriers, $\left(m^{-3}\right)$.

$$
\begin{aligned}
& n_{0}=\frac{n_{i}}{N_{a}} \text {, the concentration at equilibrium, }\left(m^{-3}\right) . \\
& n_{i}, \text { is the intrinsic carrier, }
\end{aligned}
$$

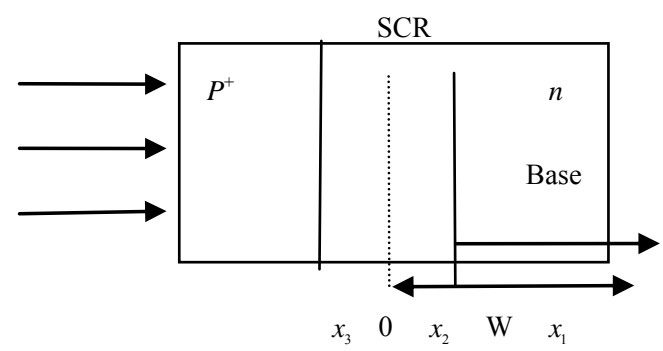

Figure 1. A model for the considered cell.

$N_{a}$, is the concentration of the acceptor atoms,

$\tau$, the recombination life time (sec) $D_{n}$, the diffusion coefficient $\left(\frac{m^{2}}{\sec }\right) G(\lambda, x)$, is the source function defined as [1]

$$
\begin{aligned}
G(\lambda, x) & =\Phi(\lambda) \alpha(1-R) \exp (-\alpha x) \\
& =\mu(\lambda) \exp (-\alpha x)
\end{aligned}
$$

where,

$$
\begin{aligned}
& \phi(\lambda),\left(\mathrm{m}^{-2} \mathrm{sec}^{-1}\right) \text { the incident solar photon flux [17]. } \\
& \alpha,\left(\mathrm{m}^{-1}\right) \text { absorption coefficient. } \\
& R, \text { the reflection coefficient at the front surface. }
\end{aligned}
$$

$$
\mu(\lambda)=\phi(\lambda) \alpha(1-R)
$$

Equation (1) is subjected to the following conditions:

$$
\begin{aligned}
& \text { at } x=0-\left.D_{n} \frac{\partial\left(n(x, t)-n_{0}\right)}{\partial t}\right|_{x=0} \\
& =\left.s_{i}\left(n(x, t)-n_{0}\right)\right|_{x=0} \rightarrow(\mathrm{i}) \\
& \text { at } x=0, t=\left.0 \quad n(x, t)\right|_{x=0, t=0} \\
& =n_{0} \exp \left[\frac{q V_{a}}{k T}\right] \rightarrow(\mathrm{ii})
\end{aligned}
$$

(ii) is a starting (initial) condition.

$$
\text { at } x=\left.W \quad n(x, t)\right|_{x=w}-n_{0}=0 \rightarrow(\text { iii })
$$

where $x=0$ is an arbitrary plane in the depletion layer $s_{i}$, the recombination velocity between the charge carriers at the boundary $x=0$.

$V_{a}$ is the applied bias voltage.

$W$, is the width of the base region.

Let the solution of Equation (1) be in the form:

$$
n(x, t)=n_{0}-v(x, t) \exp \left(\frac{-t}{\tau}\right)
$$

Substitute Equation (3) into Equation (1) to get:

$$
\frac{\partial^{2} v(x, t)}{\partial x^{2}}-\frac{1}{D_{n}} \frac{\partial v(x, t)}{\partial t}=\frac{G(\lambda, x)}{D_{n}} \exp \left(\frac{t}{\tau}\right)
$$

The Fourier separation of variables method is used to find the solution of the homogeneous part. This is ob- 
tained in the form:

$$
v_{c}(x, t)=A \exp \left(D_{n} \Gamma^{2} t\right) \exp (-\Gamma x)
$$

where, $A, \Gamma$ are parameters.

While the particular solution can be obtained using the inverse differential operator.

Equation (4) can be rewritten in the form:

$$
v_{p}=\frac{1}{D_{x}^{2}-\frac{1}{D_{n}} D_{t}}\left[\frac{\mu \exp (-\alpha x)}{D_{n}} \exp \left(\frac{t}{\tau}\right)\right]
$$

This gives the solution in the form:

$$
V_{p}(x, t)=\frac{\tau G(\lambda, x) \exp \left(\frac{t}{\tau}\right)}{\alpha^{2} D_{n} \tau-1}
$$

Thus, the general solution can be written in the form:

$$
\begin{aligned}
& v(x, t)=A \exp \left(D_{n} \Gamma^{2} t\right) \exp (-\Gamma x) \\
& +\frac{\tau G(\lambda, x) \exp \left(\frac{t}{\tau}\right)}{\alpha^{2} D_{n} \tau-1}
\end{aligned}
$$

Substituting $v(x, t)$ into Equation (3) one gets the required expression for $n(x, t)$ in the form:

$$
n(x, t)=n_{0}-A \exp (\Omega t) \exp (-\Gamma x)-\frac{\tau G(\lambda, x)}{\alpha^{2} D_{n} \tau-1}
$$

where,

$$
\Omega=D_{n} \Gamma^{2}-\frac{1}{\tau}
$$

To find $A, \Gamma$ let us apply the boundary conditions. This gives:

$$
\begin{aligned}
& A=\exp (-\Omega t) \frac{\mu \tau\left[D_{n} \alpha-s_{i}\right]}{\left[s_{i}-D_{n} \Gamma\right]\left[\alpha^{2} D_{n} \tau-1\right]} \\
& \Gamma=\frac{\mu \tau\left[D_{n} \alpha-s_{i}\right]}{D_{n}\left[f_{0}\left[\alpha^{2} D_{n} \tau-1\right]+\tau \mu\right]}+\frac{s_{i}}{D_{n}}
\end{aligned}
$$

where,

$$
f_{0}=n_{0}\left[\exp \left(\frac{q V_{a}}{k T}\right)-1\right]
$$

Finally, one gets the solution in the form:

$$
\Delta n(x, t)=f_{0} \mathrm{e}^{-\Gamma x}+\frac{\mu \tau}{\alpha^{2} D_{n} \tau-1}\left[\mathrm{e}^{-\Gamma x}-\mathrm{e}^{-\alpha x}\right]
$$

where,

$$
\Delta n(x, t)=n(x, t)-n_{0}
$$

The boundary condition (iii) makes it possible to get the equation for $S_{i}$ in the form:

$$
\left(\frac{s_{i}}{D_{n}}-\alpha\right) w=\frac{\chi \ln \chi}{\chi-1}
$$

where,

$$
\chi=\frac{f_{0}\left[\alpha^{2} D_{n} \tau-1\right]}{\mu \tau}+1
$$

Discussing the order of magnitude of different physical quantities included in expression (16) one can get the following results:

$$
|\chi|=\frac{f_{0}}{\mu \tau} \succ \succ 1 \quad \frac{s_{i}}{D_{n}} \succ \succ \alpha
$$

This gives:

$$
\tau=\frac{f_{0}}{\mu} \mathrm{e}^{-\frac{S_{i} w}{D_{n}}}
$$

Equations (15) and (17) are very important expressions since they establish a relation between the recombination life time and the recombination velocity for the given parameters.

\section{The Efficiency}

In order to estimate the efficiency, one has to compute the charge current $J_{S C}$ according to the relation:

$$
J_{S C}=J_{n}=q D_{n} \frac{\partial n(x, t)}{\partial x}
$$

$J_{S C}$ is obtained in the form:

$$
\begin{aligned}
J_{n}= & -q D_{n} \Gamma \frac{\left[f_{0}\left[\alpha^{2} D_{n} \tau-1\right]+\tau \mu\right]}{\alpha^{2} D_{n} \tau-1} \exp (-\Gamma x) \\
& +\frac{q D_{n} \alpha \tau G(\lambda, x)}{\alpha^{2} D_{n} \tau-1}
\end{aligned}
$$

The efficiency $\eta$ is expressed through the relation:

$$
J_{s c}=\eta=\frac{F F V_{O C} J_{S C}}{P_{\text {in }}}
$$

where,

$F F$ the fill factor.

$V_{O C}$ the open circuit voltage.

$J_{S C}$ the short circuit current.

$P_{\text {in }}$ the input solar power absorbed at the front surface of the solar device.

The open circuit voltage $V_{O C}$ is given as [18].

$$
V_{O C}=\frac{k T}{q} \ln \left(\frac{J_{S C}}{J_{0}}+1\right)
$$

where,

$$
k=1.38 \times 10^{-23} \frac{\mathrm{J}}{\mathrm{K}} \text { is Boltzmann constant. }
$$


$T,{ }^{0} \mathrm{~K}$ is the absolute temperature.

$J_{0}, \frac{A}{m^{2}}$ is the diode saturation current density.

$q=1.6 \times 10^{-19} \mathrm{C}$ is the charge of an electron.

The value of $J_{0}$ is expressed as [19]

$$
J_{0}=A T^{3} \exp \left[\frac{-E_{g}}{k T}\right]
$$

where, $A$ is the ideality factor. It is taken as unity for simplification.

$E_{g}$ is the energy gap expressed as [20].

$$
E_{g}(T)=E_{g}(0)-\frac{a T^{2}}{(T+b)}
$$

where, $a$ and $b$ are parameters.

\section{Computations}

As an illustrative example, computations for a silicon $p-n$ solar cell are given concerning the above mentioned functions.

For a silicon solar cell [20]:

$$
\begin{aligned}
& a=4.73 \times 10^{-4} \mathrm{eV} / \mathrm{K}, \quad b=636^{0} \mathrm{~K} \\
& E_{g}(0)=1.17 \mathrm{eV}, \quad T=300^{0} \mathrm{~K}
\end{aligned}
$$

The following values for further parameters are also considered $[1,17,21]$ :

$$
\begin{aligned}
& (1-R)=0.678, D_{\mathrm{n}}=32.3 \times 10^{-4} \frac{\mathrm{m}^{2}}{\mathrm{sec}}, \alpha=8 \times 10^{2} \mathrm{~m}^{-1}, \\
& \mathrm{n}_{0}=1 \times 10^{10} \mathrm{~m}^{-3}, \phi=7 \times 10^{19} \frac{1}{\mathrm{~m}^{2} \mathrm{sec}}
\end{aligned}
$$

1) The recombination life time as a function of the recombination velocity is computed first with " $W$ " as a parameter, then for $V_{a}$ as a parameter. The obtained results are illustrated graphically in Figures 2 and 3. It is clear that as the recombination velocity increases the recombination life time decreases.

2) The relation between $\Delta n(x, t)$ and " $x$ " for following parameters: $V_{a}=0.40$ Volts,$s_{i}=20 \mathrm{~m} / \mathrm{sec}$ and $\tau=0.41 \times 10^{-6} \mathrm{sec}$, is illustrated graphically in Figure 4. It is clear that the function $\Delta n(x, t)$ decreases with " $x$ " through the cell.

3) The current density " $J$ " and the efficiency $\eta$ is computed. The following values of the applied voltage $V_{a}$ are considered as parameters: $0.51,0.52,0.53,0.54$ volts.

The obtained values are given in Table $\mathbf{1}$ and are illustrated graphically in Figure 5.

\section{Conclusions}

The obtained results reveal that:

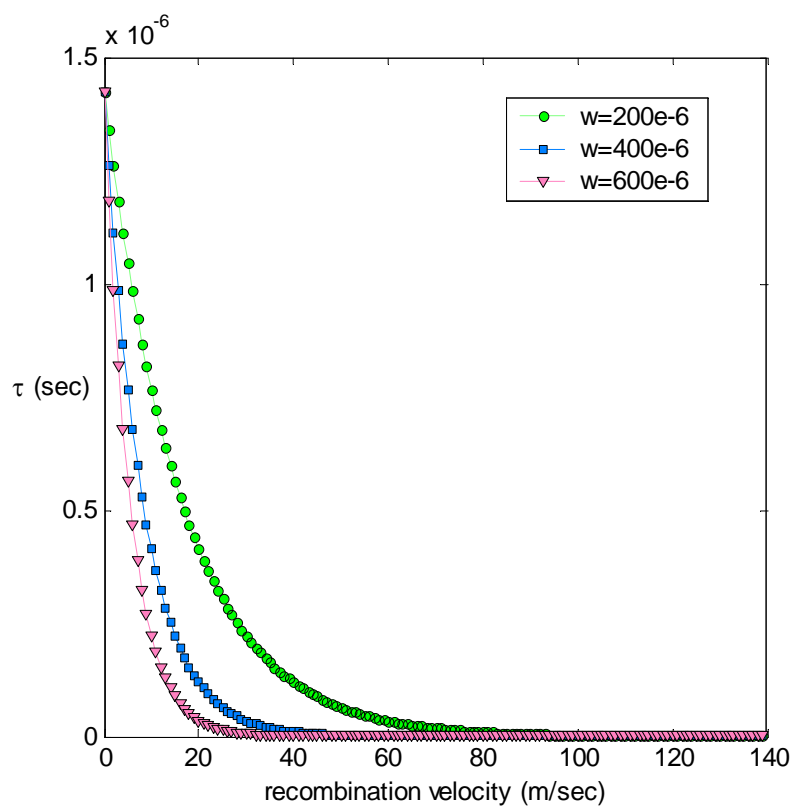

Figure 2. A relation between the recombination life time and the recombination velocity for " $W$ " as a parameter.

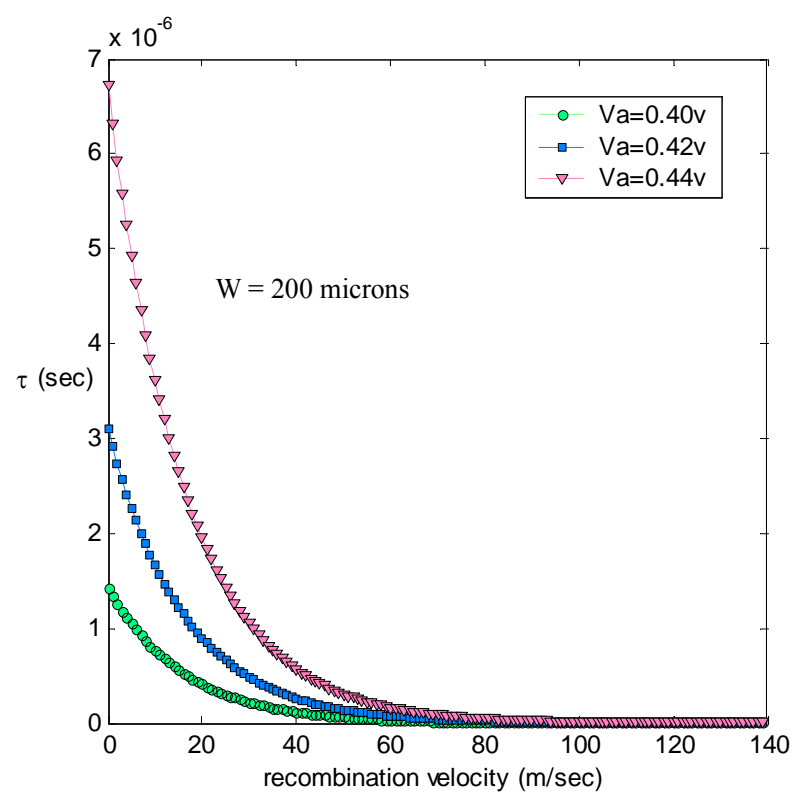

Figure 3. A relation between the recombination life time and the recombination velocity for $V_{a}$ as a parameter.

1) The recombination velocity $s_{i}$ does affect the current density through the depletion layer. It decreases with the increase of $s_{i}$. This in turns leads to the decrease in the efficiency.

2) Taking the bias voltage $V_{a}$ as a parameter. One finds that for a certain recombination velocity the applied voltage $V_{a}$ has positive effect on the efficiency.

The efficiency increases with $V_{a}$ since $J_{S C}$ increases with $V_{a}$. 


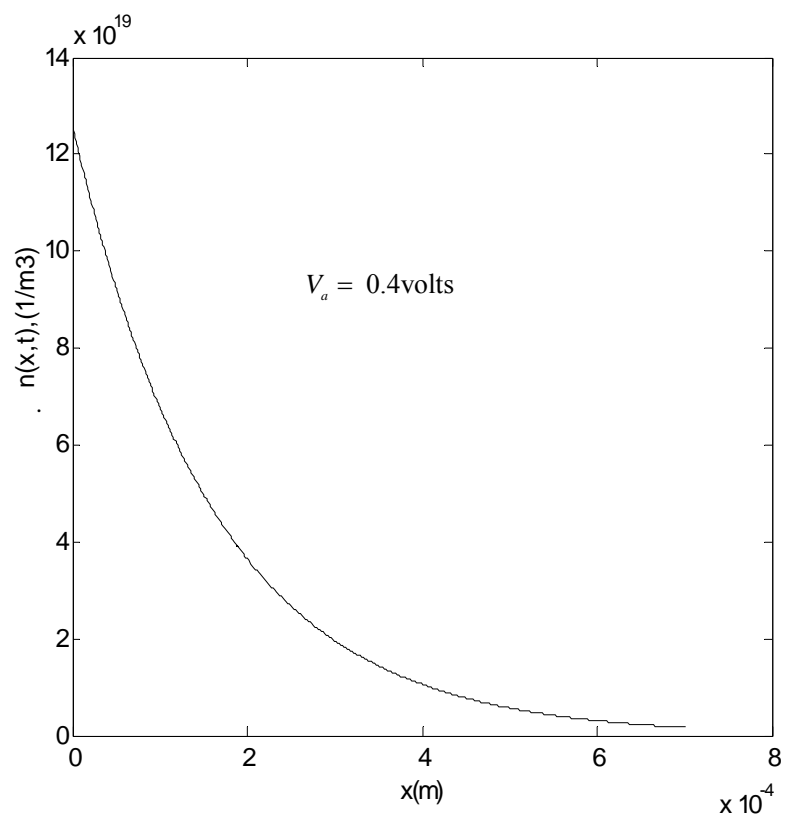

Figure 4. A between $\Delta n(x, t)$ and " $x$ " through the cell.

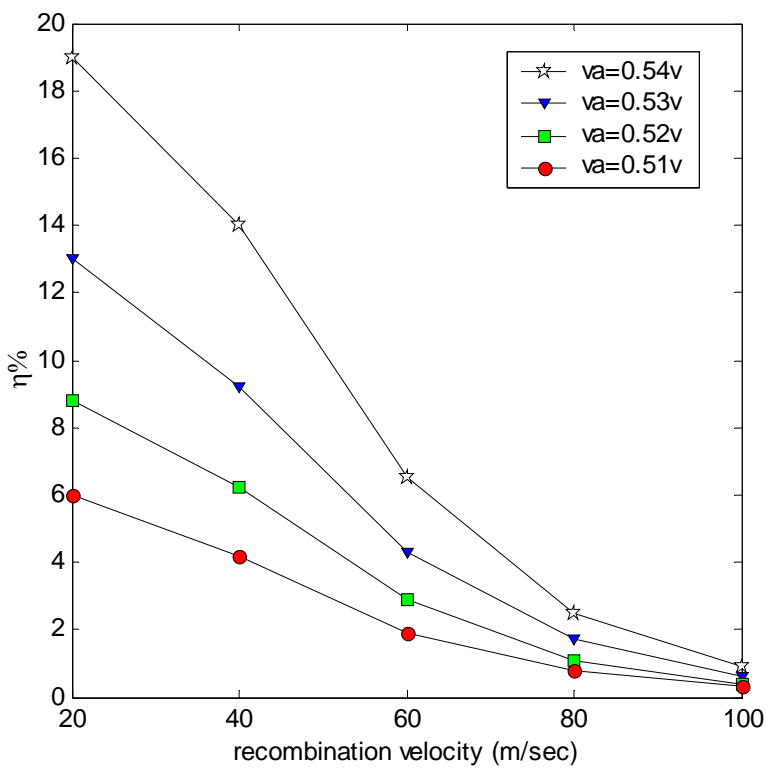

Figure 5. The efficiency $\eta$ of a silicon solar cell as a function of the recombination velocity $s_{i}$.

Table 1. The current density " $J$ " and the efficiency " $\eta$ " as a function of the recombination velocity $s_{i}(\mathrm{~m} / \mathrm{sec})$ with the applied voltage as parameter.

\begin{tabular}{|c|c|c|c|c|c|c|c|c|}
\hline & $V_{a}=0.51$ volts & & $V_{a}=0.52$ volts & & $V_{a}=0.53$ volts & & $V_{a}=0.54$ volts & \\
\hline$s_{i}(\mathrm{~m} / \mathrm{sec})$ & $J_{s c}\left(\mathrm{~A} / \mathrm{m}^{2}\right)$ & $\eta \%$ & $J_{s c}\left(\mathrm{~A} / \mathrm{m}^{2}\right)$ & $\eta \%$ & $J_{s c}\left(\mathrm{~A} / \mathrm{m}^{2}\right)$ & $\eta \%$ & $J_{s c}\left(\mathrm{~A} / \mathrm{m}^{2}\right)$ & $\eta \%$ \\
\hline 20 & 2.53 & 6. & 3.68 & 8.8 & 5.29 & 13 & 7.5 & 19 \\
\hline 40 & 1.77 & 4.2 & 2.6 & 6.2 & 3.83 & 9.2 & 5.63 & 14 \\
\hline 60 & 0.85 & 1.9 & 1.26 & 2.9 & 1.85 & 4.3 & 2.73 & 6.5 \\
\hline 80 & 0.35 & 0.8 & 0.51 & 1.1 & 0.75 & 1.7 & 1.1 & 2.5 \\
\hline 100 & 0.13 & 0.3 & 0.19 & 0.4 & 0.28 & 0.6 & 0.41 & 0.9 \\
\hline
\end{tabular}

3) For $\left(s_{i}=0\right)$ computation revealed that the recombination life time is not affected by the width " $w$ " of the cell. But it is positively affected by the applied voltage.

\section{REFERENCES}

[1] A. S. Bouazzi, M. Abaab and B. Rezig, "A New Model of Very High Efficiency Buried Emitter Silicon Solar Cell," Solar Energy Materials \& Solar Cells, Vol. 46, No. 1, 1997, pp. 29-41.

[2] O. Kurniawan and K. S. Vincent, "An Analysis of the Factors Affecting the Alpha Parameter Used for Extracting Surface Recombination Velocity in EBIC Measurements," Solid State Electronics, Vol. 50, No. 3, 2006, pp. 345-354. doi:10.1016/j.sse.2006.01.006

[3] C. L. Garrido, D. Stolik, J. Rodriguez and A. Morales, "Influence of Carrier Recombination in the Space Charge Region on Minority Carrier Life Time in the Base Region of Solar Cells," Solar Energy Materials \& Solar Cells, Vol. 57, No. 3, 1999, pp. 239-247.
doi:10.1016/S0927-0248(98)00174-3

[4] K. Chakabarty and S. Nsingh, "Depletion Layer Resistence and Its Effect on I-V Characteristics of Fully-andPartially Illuminated Silicon Solar Cells," Solid state electronics, Vol. 39, No. 4, 1966, pp. 577-581. doi:10.1016/0038-1101(96)00179-7

[5] A. B. Kaiser, "Electronic Transport Properties of Conducting Polymers and Carbon Nanotubes," Progress in Physics, 64, No. 1, 2001, pp. 1-49. doi:10.1088/0034-4885/64/1/201

[6] A Cuevas, P. A. Basore, M. C. Girouit and C. Dubois, "Surface Recombination Velocity of Highly Doped nType Silicon," Journal of Applied Physics, Vol. 80, No. 6, 1966, p. 3370. doi:10.1063/1.363250

[7] P. Kittidachachan, T. markvart, D. M. Bagnall, R. Greef, and G. J. Ensell, "A Detailed Study of $p-n$ Junction Solar Cells by Means of Collection Efficiency," Solar Energy Materials and Solar Cells, Vol. 91, No. 2-3, 2007, pp. 160-166. doi:10.1016/j.solmat.2006.08.002

[8] S. Daliento, L. Mele, E. Bobeico, L. Lancellotti and P. 
Morrillo, "Analytical Modeling and Minority Current Measurements for the Determination of the Emitter Surface Recombination Velocity in Silicon Solar Cells," Solar Energy Materials \& Solar Cells, Vol. 91, No. 8, 2007, pp. 707-713. doi:10.1016/j.solmat.2006.12.007

[9] F. A. Lindholm, L. Juinj and A. N. Schel, "Determination of Life Time And Surface Recombination Velocity of $p$ n-Junction Solar Cells And Diodes by Observing Transients," IEEE Transactions on Electron Devices, Vol. 34, No. 2, 1987, pp. 277-285.

[10] S. N. Singh and P. K. Singh, "Modeling of MinorityCarrier Surface Recombination Velocity at Low-High Junction of an $p-n$ Silicon Diode," IEEE Transaction on Electron Devices, Vol. 38, No. 2, 1991, pp. 337-343. doi:10.1109/16.69915

[11] S. S. de, A. K. Ghosh, M. Bera, A. Hajra and J. C. Halder, "Influence of Built-in-Potential on the Effective Surface Recombination Velocity for Heavily Doped High-Low Junction," Physics B: Physics of Condensed Matter, Vol. 228, No. 3-4, 1966 , pp. 363-368.

[12] S. Kumar, P. K. Singh, G. S. Chilana and S. R. Dhariwal, "Generation and Recombination Life Time Measurement in Silicon Wafers Using Impedance Spectroscopy," Semiconductor Science and Technology, Vol. 24, No. 9, 2009, pp. 1-8, Article ID: 095001.

[13] J. F. Gibbons, "Semiconductor Electronics," McGrawHill, New York, 1966, Chapter 6.
[14] M. Saad and A. Kassis, "Effect of Interface Recombination on Solar Cell Parameters," Solar Energy Materials \& Solar Cells, Vol. 79, No. 4, 2003, pp. 507-517. doi:10.1016/S0927-0248(03)00101-6

[15] M. K. El-Adawi and N. S. Al-Shameri, "On the Depletion Layer," Material Science Research India, Vol. 6, No. 1, 2009, pp. 73-78.

[16] H. Awaki, K. Tachibana, Y. Tamai, K. Yamamoto, S. Kitamoto and M. Tsujimoto, "A Novel Method to Estimate the Thickness of the Depletion Layer of an X-Ray CCD," Nuclear Instruments \& Methods in Physics Research (Section A), Vol. 495, 2002, pp. 232-239.

[17] M. K. El-Adawi and N. S. Al-Shameri, "The Efficiency of the Solar Converter as a Function of the Doping Degrees and the Incident Solar Spectral Photon Flux," Canadian Journal on Scientific and Industrial Research, Vol. 3, No. 3, 2012, pp. 112-122.

[18] S. M. Sze, "Physics of Semiconductor Devices," Wiley, New York, 1981.

[19] M. A. Green, "Solar Cells," Prentice-Hall, Englewood Cliffs, 1982.

[20] R. F. Pierret, "Semiconductor Devices Fundamentals," Addison-Wesley Publishing Company, Boston, 1996.

[21] A. Bhattacharyya and B. G. Streetman, "Dynamics of Pulsed $\mathrm{CO}_{2}$ Laser Annealing of Silicon," Journal of Applied Physics, Vol. 14, No. 5, 1981, pp. 67-72. 